\title{
DESENVOLVIMENTO DE ANEMÔMETRO DE COPOS COM ARDUINO
}

\author{
Rodolfo Martins Albuini - rodolfo.albuini@alunos.unis.edu.br* \\ Filipe de Oliveira Proença - filipe.proenca@ alunos.unis.edu.br* \\ Guilherme Reis Portilho - Guilherme.portilho@alunos.edu.br* \\ Victor Hugo Silva Lucas - victor.lucas@alunos.unis.edu.br* \\ Patrícia Werneck S. de Oliveira - paticso@yahoo.com.br*
}

RESUMO: Este artigo aborda o desenvolvimento do protótipo de um Anemômetro de copos com Arduino, elaborado a partir de uma análise de campo por meio de projetos já desenvolvidos. Este protótipo foi desenvolvido com o objetivo de medir a velocidade do vento em função do tempo, calculando, assim, se a área em estudo é adequada ou não para a demanda solicitada.

Palavras-chave(s): Anemômetro de Copos. Protótipo. Velocidade do vento.

\section{INTRODUÇÃO}

Segundo dados do Instituto Brasileiro de Geografia e Estatística (IBGE), a geração de energia eólica expandiu aproximadamente 460,9\% de 2010 a 2014, saltando de 2.177 gigawatts/hora $(\mathrm{GWh})$ para $12.210 \mathrm{GWh}$ anuais no período. Rio Grande do Norte, Ceará e Rio Grande do Sul, nessa ordem, são os estados que lideram a geração de energia elétrica com a força do vento.

Apesar de todo esse crescimento, a energia eólica corresponde a apenas 2,1\% da energia total gerada no Brasil, segundo dados da Revista Época (2016). Baseando-se nesses dados, a idealização do protótipo do Anemômetro Arduino tem como funcionalidade calcular a velocidade do vento em função do tempo em determinada área em estudo, identificando se será possível ou não realizar as demandas solicitadas.

O objetivo do presente artigo é desenvolver um protótipo de Anemômetro com Arduino Uno juntamente com o sensor Reed Switch. O anemômetro funciona como um coletor de ventos através de suas conchas, no qual a força do vento empurra as conchas de forma rotacional fazendo com que o eixo central movimente o reed switch gerando pulsos digitais que são transmitidos para o arduino, convertendo assim os resultados através de equações matemáticas e enviando-os para o display de LCD.

\section{REFERENCIAL TEÓRICO 2.1 Energia Eólica}

De acordo com Silva et al. (2019), a energia eólica é a energia cinética presente na massa de ar em movimento (vento). Essa energia renovável é obtida a partir do princípio da conversão de energia cinética de translação em energia cinética de rotação, usando turbinas eólicas ou aerogeradores. 


\subsection{Anemômetro}

De acordo com Frenzel Jr. (2015), os anemômetros são instrumentos no qual sua função é medir direção e velocidade dos ventos, sendo geralmente utilizados nas indústrias do agronegócio, em estações meteorológicas e estudos de instalação de turbinas eólicas. Esta palavra deriva do grego "anemos", que significa vento.

Os anemômetros de uso generalizado para medir velocidade de vento são formados por três ou mais conchas de formato especial montadas simetricamente estabelecendo ângulos retos em relação a um eixo vertical (ALMEIDA, 2019).

Segundo Sampaio et al. (2005, apud PEARCY et al., 1989), os anemômetros que utilizam o princípio de quantidade de movimento são os anemômetros de conchas, de deflexão e de hélices. Outros usam o princípio físico da termoeletricidade, como os anemômetros de fio quente, de termistor aquecido e de termopilhas.

De acordo com a velocidade do vento e os objetivos para a sua medição, utilizam-se anemômetros com características de construção e operações diferentes. De forma geral, o mais usado é o anemômetro de rotação (copos). Este instrumento indica a velocidade do vento unidirecional, sendo a velocidade exibida por uma equação linear (SAMPAIO, 2005).

\subsubsection{Anemômetro de rotação}

Dentre os anemômetros presentes no mercado, um dos mais tradicionais é o de rotação, segundo Koyama et al. (2007).

De acordo com Seidler et al. (2011), os anemômetros de rotação são basicamente constituídos por hélices que giram a uma velocidade correspondente à velocidade do vento que passa entre elas. São calibrados de forma que o total de voltas dadas por suas pás equivalha a uma velocidade específica, isto é, se no túnel de vento em que são ajustados a corrente de ar sopra a dez quilômetros por hora, e as pás do instrumento giram cem vezes por minuto, ele é programado para indicar $10 \mathrm{~km} / \mathrm{h}$ sempre que o anemômetro alcançar 100 rotações por minuto, e assim por diante.

Conforme Sampaio et al. (2005), as conchas (geralmente 3) são fixadas nas extremidades de hastes presas a um eixo central vertical e girável formando ângulos iguais entre si de $120^{\circ}$, de maneira que suas partes côncavas permaneçam sempre voltadas no mesmo sentido para que girem com a pressão do vento em torno do eixo central.

A vantagem de se utilizar esse anemômetro é o baixo custo, porém existe a desvantagem de possuir peças móveis que impedem medidas precisas devido ao atrito com o ar. Outra desvantagem é a necessidade da velocidade inicial ter uma força capaz de vencer a inércia das conchas ou hélices (KOYAMA et al., 2007).

\subsection{Arduino UNO}

Segundo Frizzarin (2016), o Arduino UNO é uma placa micro controladora que possui 14 pinos de entrada/saída digital, 6 pinos de entrada analógica, um cristal de quartzo de $16 \mathrm{MHz}$, uma conexão USB, um conector de energia, um conector ICSP e um botão de reset. Possui $32 \mathrm{~KB}$ de memória em flash e sua tensão de alimentação é de $5 \mathrm{~V}$, podendo ser alimentado por fonte externa 
Figura 1: Arduino uno

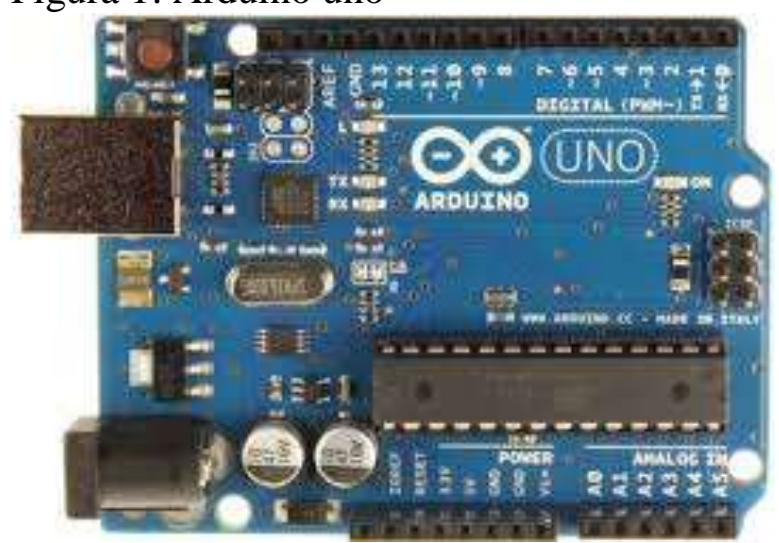

Fonte: Google imagens (2019).

A Figura 1 demostra a placa Arduíno Uno que serve como plataforma de desenvolvimento de protótipos a partir de prototipagem eletrônica de hardware livre.

\subsection{Reed Switch}

Os reed switches ou interruptores de lâminas são definidos como dispositivos constituídos por um bulbo de vidro no interior do qual existem lâminas flexíveis feitas de materiais que podem sofrer a ação de campos magnéticos. Geralmente são utilizados como sensores em muitas aplicações na robótica, mecatrônica e automação (BRAGA, 2019), conforme a figura 2.

Figura 2: Reed Switch

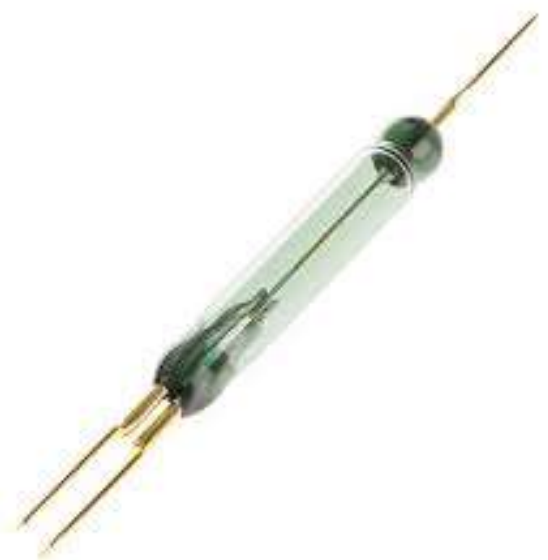

Fonte: Google imagens (2019).

Na figura 2 é ilustrado o Reed Switch que é formado por um bulbo de vidro com uma lâmpada em seu interior, que em ação do campo magnético se movimenta de forma a fornecer um 
contato fechado ou aberto. Ao aproximar-se o reed a um ímã, um campo magnético induz as lâminas a se unirem, assim fechando um contato elétrico.

\section{METODOLOGIA}

A tabela 1 mostra os equipamentos utilizados na construção do protótipo e suas especificações, que estão disponíveis no mercado.

Foram utilizados para o desenvolvimento do protótipo os itens da tabela 1, sendo o Arduino UNO a peça fundamental para a leitura do código da programação e transmissão do código para os diversos elementos do projeto.

Tabela 1: Descrição dos equipamentos para a montagem do protótipo.

\begin{tabular}{ccc}
\hline Materiais utilizados & Quantidade & Descrição \\
\hline Anemômetro & 01 & Alumínio \\
Protoboard & 01 & 400 pontos \\
Arduino & 01 & $\mathrm{UNO}$ \\
Cabo de Energia & 01 & $5,5 \times 2,1 \mathrm{~mm}$ \\
Bateria & 01 & Alcalina $9 \mathrm{~V}$ \\
Jumpers & 10 & Macho-macho \\
Resistores & 01 & $1000 \Omega$ \\
Resistores & 02 & $220 \Omega$ \\
Display & 01 & LCD $16 \times 2 \mathrm{~cm}$ \\
Reed Switch & 01 & Leitor de Rotações \\
\hline
\end{tabular}

Tabela 01 (Fonte: Próprio autor)

O presente artigo foi elaborado através de uma análise de campo por meio de projetos já desenvolvidos. Este protótipo foi desenvolvido com o intuito de medir a velocidade do vento em função do tempo, calculando, assim, se a área em estudo é adequada ou não para a demanda solicitada. Para esta finalidade, foram analisados artigos e livros dispostos no acervo das Faculdades Integradas de Cataguases (FIC/UNIS). Já no banco de dados do Google Acadêmico foram consultados artigos científicos, revistas científicas, livros, sites de universidades federais, sites nacionais.

A implementação do projeto inclui a utilização do programa na linguagem C no Arduino, onde está contida toda a sua funcionalidade. Após esta etapa, transfere-se todo o programa 
desenvolvido para o microcontrolador Arduino UNO, cuja finalidade é fazer a leitura técnica das funções programadas e comunicar aos aparelhos acoplados as funções determinadas no programa.

No desenvolvimento do protótipo foram usados materiais específicos adaptados ao projeto em questão. Fez-se uso de um anemômetro de corpo de alumínio no modelo de copos com o tamanho diametral de $76 \mathrm{~mm}$, arduino Software em linguagem de programação C, microcontrolador arduino UNO, sensor reed switch, display LCD 16x2, jumpers macho-macho, bateria de 9V, conforme demonstrado pela Figura 3.

Figura 3: Montagem do circuito na protoboard

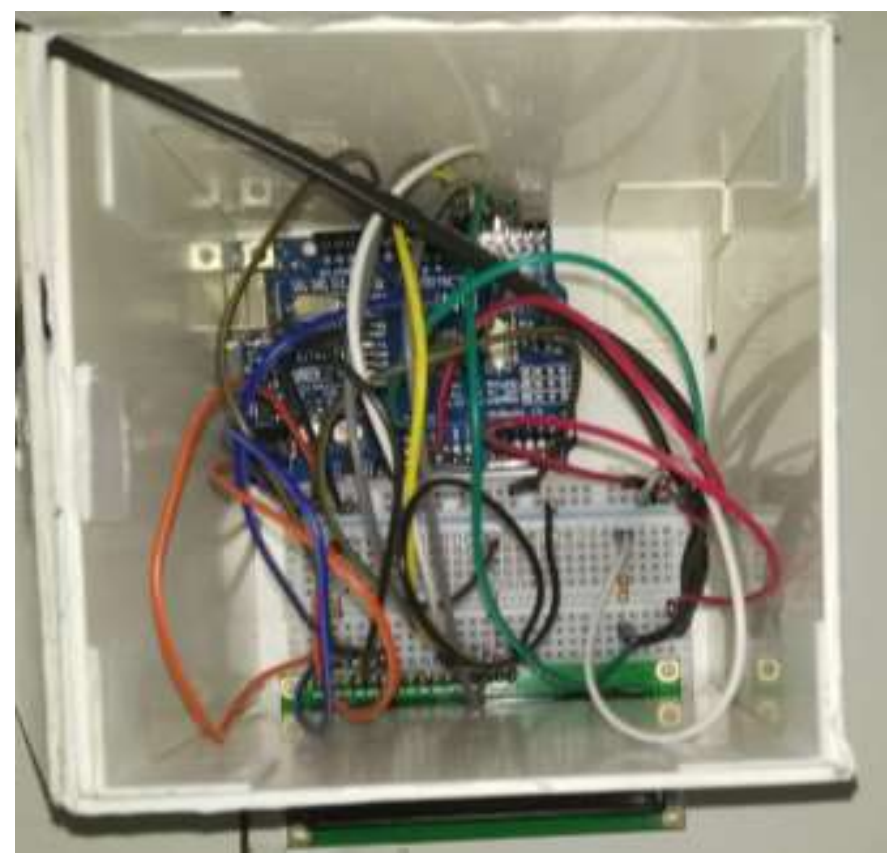

Fonte: Autores (2018).

Conforme a Figura 3, jumpers macho-macho foram utilizados interligados entre si e na protoboard, os quais foram conectados ao sensor Reed Switch pelas portas GND e VCC, simultaneamente acopladas ao Arduino UNO pelos pinos VCC e GND e pela entrada 2. A conexão dos jumpers nos pinos do display LCD foram feitas entre as entradas 3, 4, 5, 6, 12 e 13 do Arduino. Logo, o próximo passo demonstra as conexões do VCC e GND do display na protoboard. Em seguida, ocorre a conexão do cabo USB ao Arduino, transferindo a programação feita no software para a memória do Arduino, conforme a Figura 4. Na última etapa ocorre a conexão do cabo de energia juntamente com a bateria $9 \mathrm{~V}$ à placa Arduino. 
Figura 4: Sistema com o sensor e o display LCD

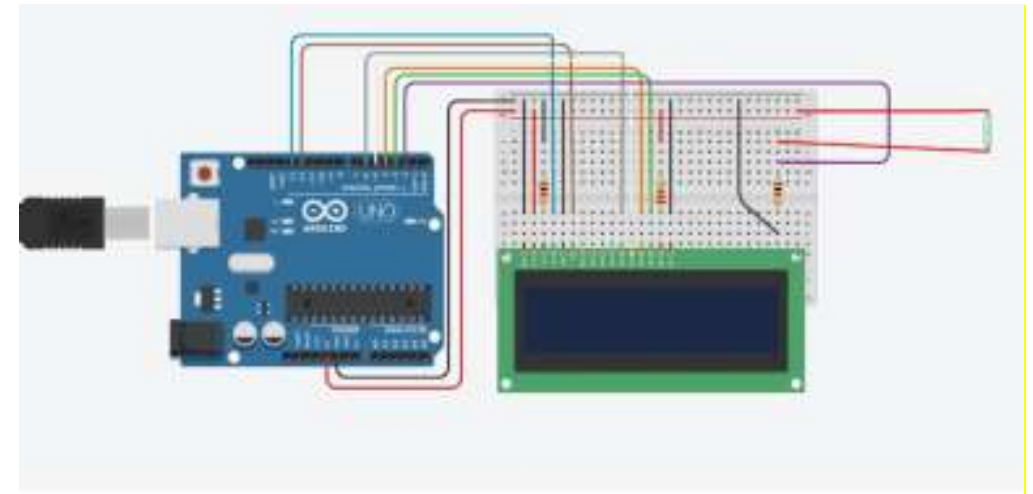

Fonte: Autores (2018).

A figura 4 ilustra as ligações entre os jumpers macho-macho com a protoboard, o Arduino e o display LCD. Também ilustra a conexão do cabo USB no Arduino para a transferência da programação.

Finalizada a montagem do protótipo e implementada a programação, foi realizado o teste em uma sala fechada com o ventilador da marca ARNO, modelo VF40 com 126 W de potência, no indicador de velocidade número três paralelamente ao anemômetro. O visor do display demonstra a cada 5 segundos os resultados obtidos em $\mathrm{Km} / \mathrm{h}$ e m/s conforme figura 5 .
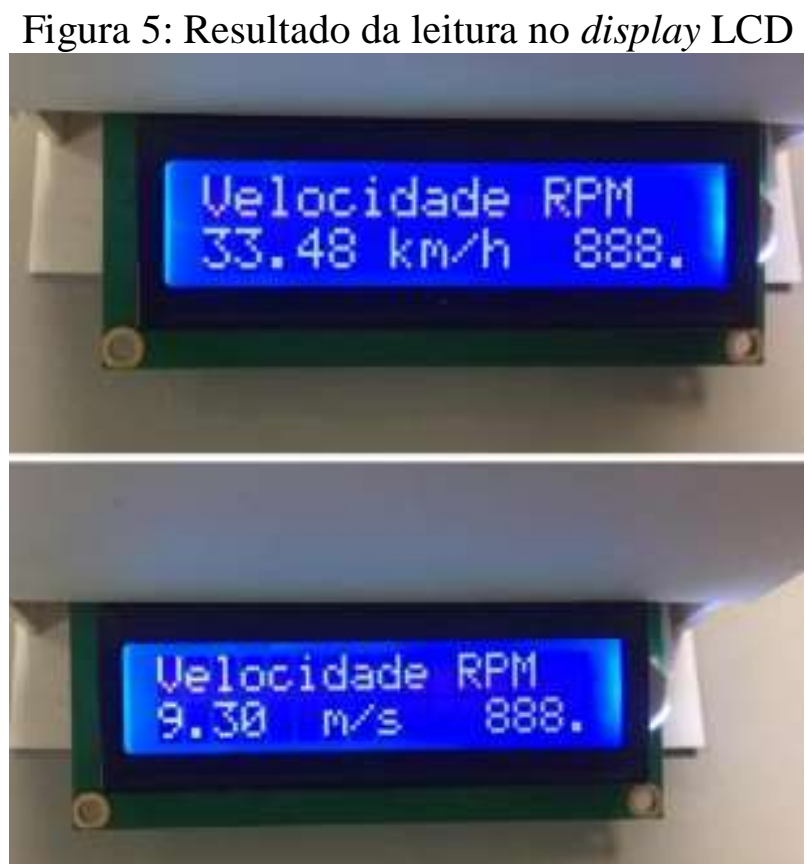

Fonte: Autores (2018). 
A Figura 5 apresenta o resultado da medição do vento a cada 5 segundos de acordo com o teste realizado. Foram computados os valores de $33,48 \mathrm{Km} / \mathrm{h}$ e $9,30 \mathrm{~m} / \mathrm{s}$.

\section{RESULTADOS E DISCUSSÕES}

O desenvolvimento do protótipo tem como objetivo calcular a velocidade do vento em relação ao tempo.

$\mathrm{O}$ anemômetro funciona como um coletor de ventos através de suas conchas, no qual o vento passa pelas conchas movimentando-as de forma rotacional e fazendo com que o eixo central gire o reed switch gerando pulsos digitais que são transmitidos para o arduino, convertendo assim os resultados através de equações matemáticas e enviando-os para o display de LCD, conforme figura 6.

Figura 6. Anemômetro Arduino

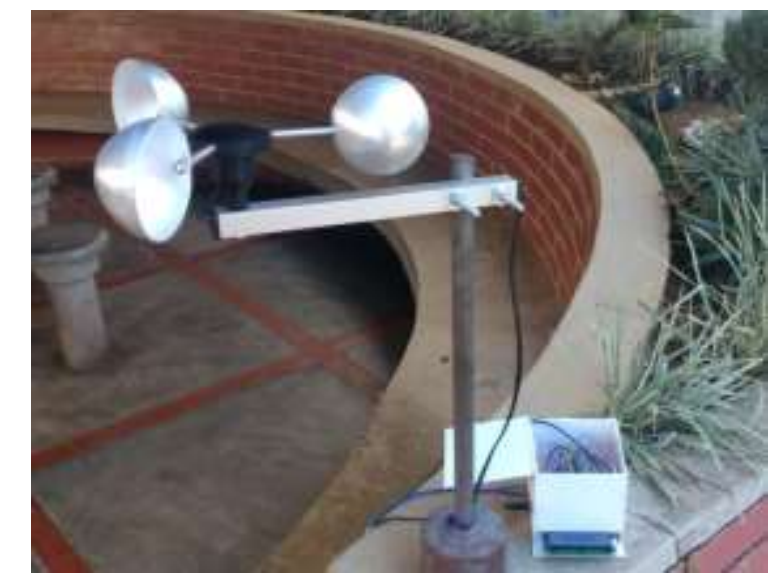

Fonte: (Autores, 2019). estudos.

A figura 6 demonstra o protótipo finalizado, pronto para ser utilizado para testes e

Depois de implementado o programa e finalizada a montagem do projeto, concluiu-se que o tempo ideal para as medições é acima de 5 segundos, pois com tempos menores do que esse o "Reed Switch" (leitor de rotações) não consegue medir ventos abaixo de $30 \mathrm{~km} / \mathrm{h}$, já com o tempo de 5 segundos é possível medir até no mínimo $2 \mathrm{~km} / \mathrm{h}$.

\section{CONSIDERAÇÕES FINAIS}

A crescente demanda nacional de energia elétrica somada aos grandes impactos causados ao meio ambiente devido à queima de combustíveis fósseis utilizados na complementação da matriz energética brasileira, bem como os desastres naturais ocasionados pela força excessiva dos ventos são os principais motivos que levaram ao desenvolvimento deste protótipo. Nosso protótipo tem como objetivo calcular a velocidade do vento em relação ao tempo. 
O protótipo é ligado a uma bateria externa de 9 volts, cuja função é alimentar o sistema. O desenvolvimento do anemômetro deu-se pelo uso de um Arduino Uno cuja programação foi desenvolvida no Arduino Software e posteriormente enviada à memória do Arduino. Através dos jumpers macho-macho conectou-se o sensor Reed Switch na Protoboard e no display LCD.

A principal contribuição do nosso protótipo é auxiliar na análise de elaboração de parques eólicos e também colaborar com estudos meteorológicos.

\section{REFERÊNCIAS BIBLIOGRÁFICAS}

ALMEIDA, S. G. Estudo de viabilidade técnica e econômica para a implantação de um sistema híbrido de geração de energia elétrica no Instituto Federal de Ciência e Tecnologia de Roraima. 2019. Disponível em: 〈https://repositorio.unesp.br/handle/11449/180745>. Acesso em: 27 de março de 2019.

BRAGA, N. C. Como funciona o Reed Switch. 2019. Disponível em:

<https://www.newtoncbraga.com.br/index.php/como-funciona/2462-art373>. Acesso em: 18 de abril de 2019.

ÉPOCA, REVISTA. Geração de energia eólica cresce $\mathbf{4 6 1 \%}$ em 4 anos, revela IBGE. 2016. Disponível em: $<$ https://epocanegocios.globo.com/Empresa/noticia/2016/06/epoca-

negocios-geracao-de-energia-eolica-cresce-461-em-4-anos-revela-ibge.html>. Acesso em: 02 de maio de 2019.

FRENZEL JR., LOUIS E. Eletrônica Moderna. 2015. Disponível em:

<https://books.google.com.br/books?id=MHDOCgAAQBAJ\&pg=PA421\&dq=jr.+louis+frenzel+ANEMOMETRO

$\&$ hl=ptBR\&sa=X\&ved=0ahUKEwibi6XZws7iAhWMo1kKHemRCBUQ6AEIKzAA\#v=onepage\&q=jr.\%20louis\%

20frenzel\%20ANEMOMETRO\&f=false $>$. Acesso em: 21 de março de 2019.

FRIZZARIN, F. B. Arduino: Guia para colocar suas ideias em prática. 2016. Disponível em:

$<$ https://books.google.com.br/books?id=FcPXCwAAQBAJ\&printsec=frontcover

$\&$ hl=pt-BR\#v=onepage \&q\&f=false>. Acesso em: 02 de maio de 2019.

KOYAMA, M. H. Desenvolvimento de um Anemômetro 3D ultra-sônico baseado em Apenas Quatro

Transdutores. 2007. Disponível em: 〈http://www.bibliotecadigital.uel.br/document/?code=vtls000151665>.

Acesso em: 06 de abril de 2019.

SAMPAIO, C. A. P. Desenvolvimento e avaliação de anemômetro de copos de fácil construção e operação.

2005. In: PEARCY et al. Plant physiological ecology; field methods and instrumentation. New York: Chapamn and Hall, 1989. Disponível em: <revistas.udesc.br/index.php/agroveterinaria/article/-download/5400/3602>. Acesso em: 26 de março de 2019.

SEIDLER, N. Estudo da energia eólica para aproveitamento em pequenos empreendimentos. 2011. Disponível em: 〈http://www.reitoria.uri.br/ vivencias/Numero_013/artigos/artigos_vivencias_13/n13_13.pdf>. Acesso em: 06 de abril de 2019.

SILVA, B. L. O. Estudo comparativo da análise de performance deTurbinas eólicas no domínio de frequência. 2019. Disponível em:

$<$ http://repositorio.ufersa.edu.br/bitstream/prefix/1560/1/BrendowLOS_ART.pdf $>$. Acesso em: 21 de março de 2019.

\section{Apêndice - Programação}

\#include <LiquidCrystal.h>

\#define PERIODO 5000

\#define RAIO 100

\#define DELAY 1000

int NUMERO_VOLTAS $=10$;

int geracao $=0$;

struct Vento \{ 
float $\mathrm{ms}$;

float $\mathrm{km}$;

int rpm;

;

typedef struct Vento Vento;

//Define os pinos que serão utilizados para ligação ao display

LiquidCrystal lcd(12, 11, 6, 5, 4, 3);

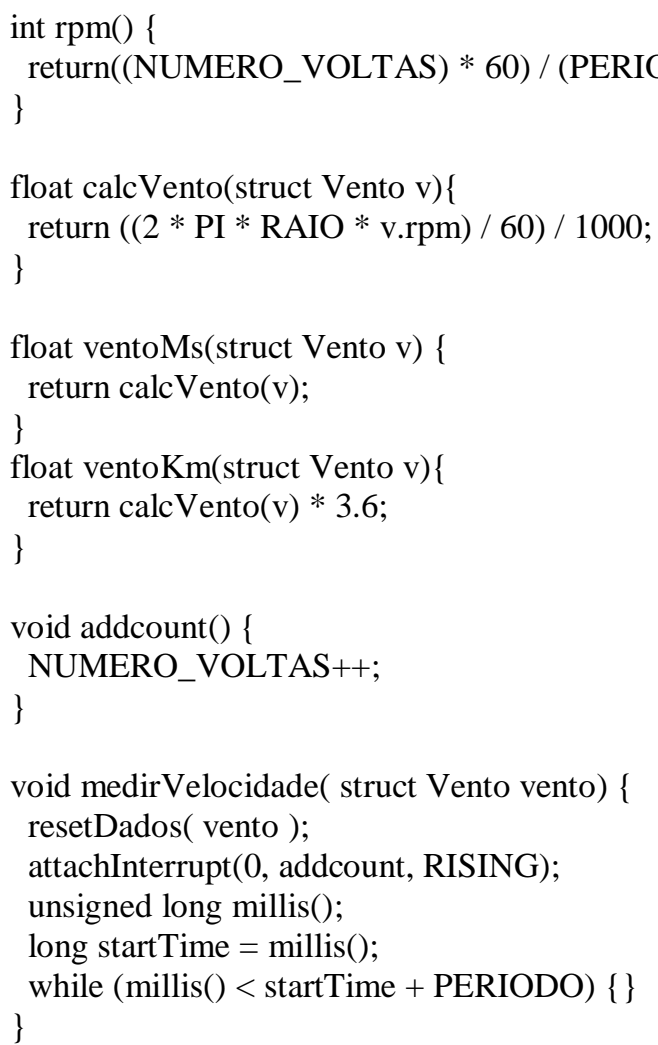




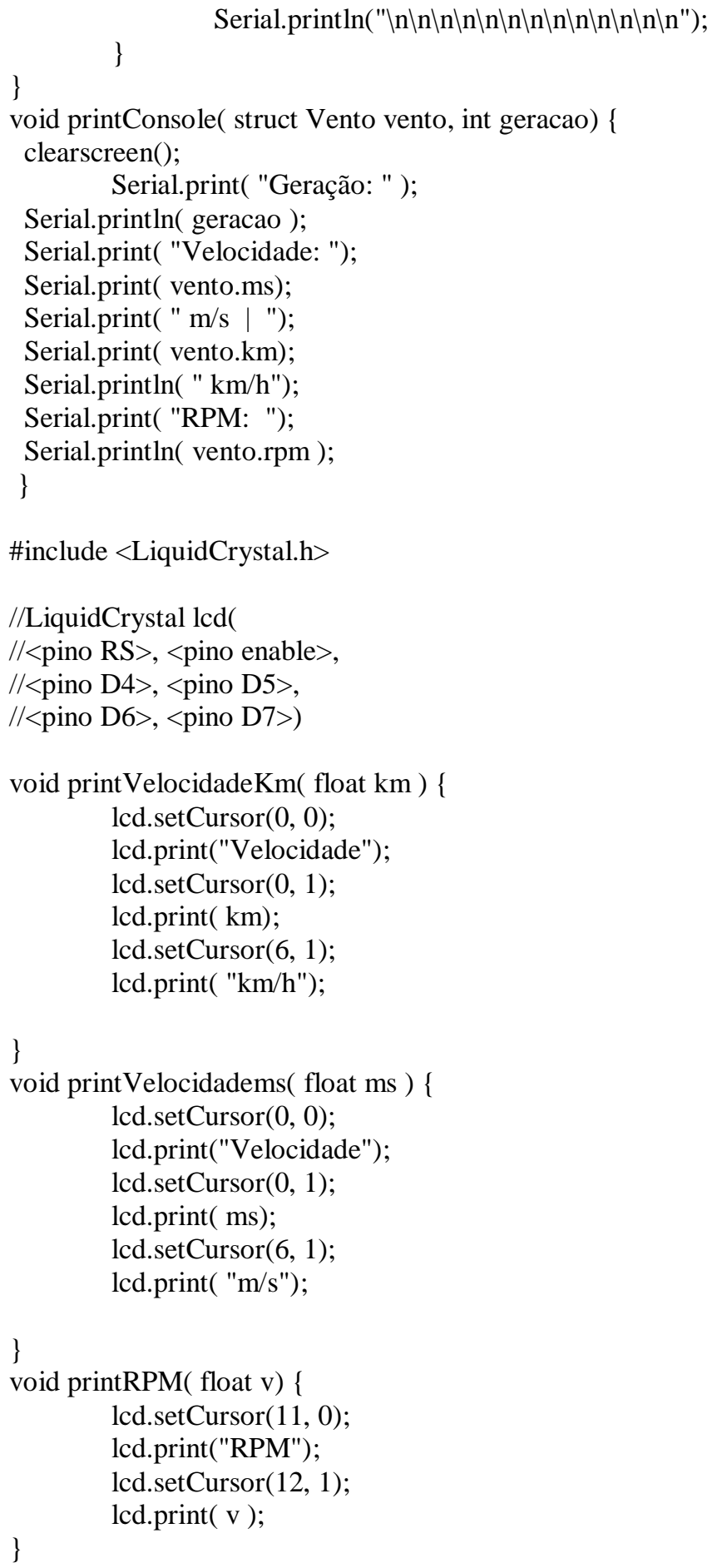

void print ( float $\mathrm{km}$, float $\mathrm{ms}$, int $\mathrm{rpm}$ ) \{

//Limpa a tela

lcd.clear();

//Posiciona o cursor na coluna 3, linha 0 ; printVelocidadeKm(km); 
ISSN 1994-0098

printRPM(rpm);

//Envia o texto entre aspas para o LCD

delay(5000);

//Rolagem para a esquerda

for (int posicao $=0$; posicao $<16$; posicao++)

\{

lcd.scrollDisplayLeft();

delay(300);

\}

lcd.clear();

printVelocidadems(ms);

printRPM(rpm);

delay (5000);

//Rolagem para a direita

for (int posicao $=0$; posicao $<16$; posicao++)

\{

lcd.scrollDisplayRight();

delay(300);

)

\}

void $\operatorname{loop}()\{$

Vento vento;

geracao++;

medirVelocidade( vento );

vento.rpm $=\operatorname{rpm}()$;

vento. $\mathrm{km}=$ vento $\mathrm{Km}$ ( vento $)$;

vento.ms $=$ ventoMs ( vento $)$;

//printConsole( vento, geracao );

print( vento.km, vento.ms, vento.rpm);

delay(DELAY);

\} 